\title{
自组装单分子层在电池中的应用展望
}

孙世刚

厦门大学化学化工学院, 福建 厦门 361005

\section{Perspective on the Application of Self-Assembled Monolayers in Batteries}

\author{
Shigang Sun \\ College of Chemistry and Chemical Engineering, Xiamen University, Xiamen 361005, Fujian Province, China. \\ Email:sgsun@xmu.edu.cn \\ Published online: September 16, 2021.
}

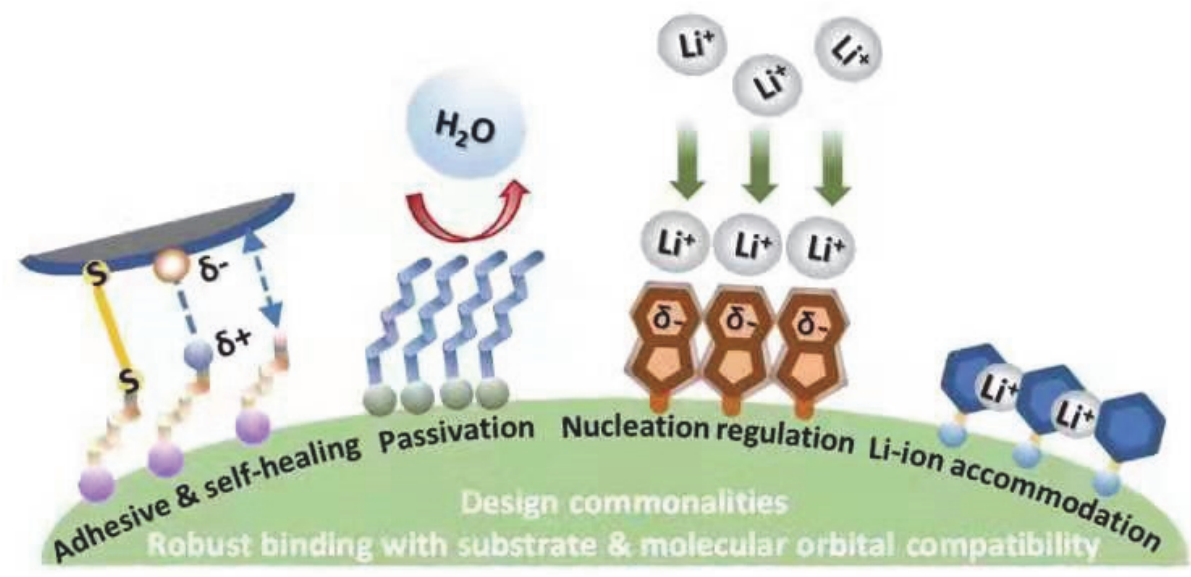

电池中的自组装单分子层的作用示意图。

高能量密度是电池技术发展的主要趋势之 一, 也是规模储能、新能源汽车等快速发展的重大 需求之一。使用高能量密度的电极活性材料是研 制高比能电池最直接的方法。然而, 高能量密度材 料通常具有不稳定的界面化学, 极大地影响电池 的循环寿命和安全性能。例如, 高镍正极材料 ${ }^{1}$ 和 金属锂负极 ${ }^{2}$ 对空气环境的敏感性, 以及二者在电 化学循环中的性能衰减行为, 都显著受到其界面 化学性质的影响。对界面进行稳定化调控成为高 比能电池发展的重要方面。一个理想的界面调控 策略, 需要能够精确调控电池界面性能的同时, 尽 可能地减少引入额外物质进入原体系, 避免对能
量密度的削弱; 同时, 该方法还应具有一定的普适 性和可规模化能力, 以适应工业化需求。

SAM (Self-assembled Monolayer)是在固体基 底和液相或气相的界面上自发吸附形成的有序单 分子层。1946年美国Zisman等3首次报道并研究了 SAM, 发现其具有自组装性、分子排布有序性, 以 及对界面的改性能力。之后SAM在防腐 ${ }^{4}$ 、润滑 5 、 感应器6、电子器件7等行业中的界面修饰中都有重 要应用。在自组装过程中, SAM分子通过化学键 与基底连接, 并形成了具有高度有序结构的分子 层8。由于修饰后的界面被尾部基团覆盖, 因此界 面的需求可以通过改变尾部基团来进行满足。由 
于SAM的生长是从无序到有序(熵减), 环境摘增的 补偿使其成为一个热力学自发过程, 这使得SAM 的修饰通常对能耗需求较低, 并且界面覆盖率较 高 ${ }^{9}$ 。可以看出, SAM 可作为一种界面改性工具, 较好地满足电池中的界面问题需求。虽然已有对 此展开研究工作的报导, 但相关内容仍然未能引 起足够关注。

为此, 中国科学院苏州纳米技术与纳米仿生 研究所沈炎宾、陈立桅研究员团队基于课题组过 去几年的研究基础上, 对电池领域中的SAMs应用 进行了系统地总结, 同时据此对SAMs在未来电池 中的应用类型与设计要点进行了展望。该篇 Perspective文章近期在线发表在 Journal of the American Chemical Society 上 $^{10}$ 。

在背景介绍中, 作者通过总结电池结构、电池 材料等参数在过去三十年间的发展历程, 提出了 界面问题是发展未来高比能电池研究焦点的观 点。另一方面, SAMs 独特的自组装特性、分子级 厚度与精确可控的化学性质, 展现了其作为解决 界面问题的可能性。文章接下来从分子结构、生长 机理及性能表征方法等方面对SAMs进行了简介。 之后作者按照不同化学电池原理(插层化学与转 化化学), 对目前SAMs在电池中的应用进展进行 了分类与总结。在插层化学类电池中, SAMs的应 用潜力主要体现在正极材料界面的稳定化修饰。 通过总结相关研究工作, 指出 SAMs能够有效提升 高 $\mathrm{Ni}$ 三元正极材料对大气气氛的稳定性, 减少正 极材料在长时间空气储存后的性能衰减。同时 SAMs 还能提高电池循环中高电压正极界面的电 化学稳定性, 减少过渡金属离子的溶出现象。某些 其他研究则涉及 SAMs 对电解质的湿润性调节以 及将SAMs本身作为插层储锂材料。在转化化学类 电池中, 较多研究聚焦于 SAMs对 $\mathrm{Li}$ 负极的界面针 化以及诱导 $\mathrm{Li}$ 在集流体表面的均匀形核两个方 面。对相关文献的总结体现了 SAMs能显著提高 $\mathrm{Li}$ 对空气环境的稳定性, 实现 $\mathrm{Li}$ 微米颗粒在空气中 的浆料刮涂。而在集流体表面构建的具有亲 $\mathrm{Li}$ 性 的SAMs能够在电镀过程中诱导 $\mathrm{Li}$ 的均匀形核, 从 而抑制枝晶生成, 这无疑为最近颇具热度的无 $\mathrm{Li}$ 负极电池的实现提供了一个新策略。SAMs在这两 类电池中的应用都充分体现了SAMs在电池中的 巨大潜力。

在对已有的文献进行归纳梳理后, 文章展望
了SAMs在更多电池界面领域的应用可能性, 包括 SAMs 在其他金属/合金负极中的界面改性能 力，如 $\mathrm{Na} 、 \mathrm{Al} 、 \mathrm{Zn}$ 负极和其他合金类负极等。利 用具有离子/电子传导能力的尾部基团, SAMs也 可用于构建或增强界面的离子/电子通路, 提高电 池电荷传递效率。在机械强度方面, 具有如氢键、 二硫键等，能够可逆性成键的SAMs分子，将有可 能提供自愈和与粘接性等功能, 提高电池内部的 机械强度与损伤修复能力。这些功能将进一步拓 宽SAMs在电池中的发展思路。

文章在最后利用理论计算分析, 以 $\mathrm{Li}$ 界面的 SAM分子结合强度, 以及不同SAMs分子结构对分 子能级轨道的影响为例, 指出 SAMs与基体的结合 强度、SAMs的电化学稳定性是未来电池界面研究 中的关键考虑因素。同时作者也指出如何在服役 过程中对SAMs进行结构维护是不可忽视的重点。 该文章为提升高能量密度电池的性能呈现了一种 极具发展前景的界面调控策略, 对SAMs在电池领 域的发展提供了前瞻性思路。

\section{References}

(1) Gu, W.; Dong, Q.; Zheng, L.; Liu, Y.; Mao, Y.; Zhao, Y.; Duan, W.; Lin, H.; Shen, Y.; Chen, L. ACS Appl. Mater. Interfaces 2020, 12, 1937. doi: 10.1021 /acsami.9b20030

(2) Kang, T.; Wang, Y.; Guo, F.; Liu, C.; Zhao, J.; Yang, J.; Lin, H.; Qiu, Y.; Shen, Y.; Lu, W.; et al. ACS Cent. Sci. 2019, 5, 468. doi: 10.1021 /acscentsci.8b00845

(3) Bigelow, W. C.; Pickett, D. L.; Zisman, W. A. J. Colloid Sci. 1946, 1, 513. doi: 10.1016/0095-8522(46)90059-1

(4) Appa Rao, B. V.; Yakub Iqbal, M.; Sreedhar, B. Corros. Sci. 2009, 51, 1441. doi: 10.1016/j.corsci.2009.03.034

(5) Watson, S.; Nie, M.; Wang, L.; Stokes, K. RSC Adv. 2015, 5, 89698. doi: $10.1039 / \mathrm{c} 5 \mathrm{ra} 17468 \mathrm{f}$

(6) Rubinstein, I.; Steinberg, S.; Tor, Y.; Shanzer, A.; Sagiv, J. Nature 1988, 332, 426. doi: 10.1038/332426a0

(7) Singh, M.; Kaur, N.; Comini, E. J. Mater. Chem. C 2020, 8, 3938. doi: $10.1039 / \mathrm{d} 0 \mathrm{tc} 00388 \mathrm{c}$

(8) Love, J. C.; Estroff, L. A.; Kriebel, J. K.; Nuzzo, R. G.; Whitesides, G. M. Chem. Rev. 2005, 105, 1103. doi: 10.1021/cr0300789

(9) Ulman, A. An Introduction to Ultrathin Organic Films; Academic Press: San Diego, CA, USA, 1991; p. 237.

(10) Yi, R.; Mao, Y.; Shen, Y.; Chen, L. J. Am. Chem. Soc. 2021, 143, 12897. doi: 10.1021/jacs.1c04416 